\title{
Association between Endoscopist Specialty and Colonoscopy Quality: Protocol for a Systematic Review and Meta-analysis
}

\author{
Nauzer Forbes ( $\nabla$ nauzer.forbes@ucalgary.ca ) \\ University of Calgary https://orcid.org/0000-0002-6966-3908 \\ Matthew Mazurek \\ University of Calgary \\ Alistair Murray \\ University of Calgary \\ Yibing Ruan \\ University of Calgary \\ Robert J Hilsden \\ University of Calgary \\ Steven J Heitman \\ University of Calgary \\ Darren R Brenner \\ University of Calgary
}

\section{Protocol}

Keywords: Colorectal cancer, screening, colonoscopy, quality

Posted Date: March 6th, 2021

DOI: https://doi.org/10.21203/rs.3.rs-281768/v1

License: (c) (i) This work is licensed under a Creative Commons Attribution 4.0 International License. Read Full License 


\section{Abstract \\ Background}

Colonoscopy is an important modality in the provision of colorectal cancer screening. Though effective and safe, the performance of screening-related colonoscopy is variable in terms of its overall quality, with endoscopist-related factors playing an important role in this variation. The purpose of this study is to systematically review the association between endoscopist specialty and colonoscopy quality and outcomes.

\section{Methods}

A comprehensive electronic search will be carried out to determine the association between endoscopist specialty and colonoscopy quality metrics and/or outcomes in adult patients undergoing colonoscopy. Two abstracters will independently determine study eligibility, assess study quality, and abstract study data. The primary outcome will be the adenoma detection rate (ADR); secondary outcomes will include cecal intubation rate (CIR), withdrawal time (WT), adverse events (AEs) and post-colonoscopy colorectal cancer (PCCRC). Rates of the above metrics and outcomes will also be compared between clinically relevant subgroups determined a priori. DerSimonian and Laird models will be used to perform metaanalyses for each outcome. Sources of heterogeneity will be explored via meta-regression analyses, if possible.

\section{Discussion}

Given the significant established variation in colonoscopy quality, endoscopist-related factors need to be explored. Our meta-analysis will address the important question of whether the specialty of the endoscopist impacts colonoscopy quality and/or outcomes.

\section{Systematic Review Registration}

\section{PROSPERO CRD42021226251.}

\section{Background}

Colorectal cancer (CRC) is a leading cause of cancer-related mortality in the United States, where approximately 150,000 associated new cases and 50,000 associated deaths are estimated in 2020 [1]. Screening is effective in reducing the incidence of and mortality from CRC [2]. Screening-related colonoscopy plays an important role in both opportunistic and programmatic screening, being capable of both detection and resection of pre-malignant adenomatous polyps [3]. Colonoscopy is also the recommended procedure following positive primary CRC screening results from other modalities. 
Unfortunately, there is widespread variation in colonoscopy quality, with a large proportion of this variation explained by both modifiable and non-modifiable endoscopist-related factors $[4,5]$.

Several quality indicators have been proposed and are currently widely supported in order to improve colonoscopy quality and reduce variations in care. These include the adenoma detection rate (ADR) and cecal intubation rate (CIR) $[6,7]$. While generally considered a safe procedure overall, colonoscopy is also associated with rare but potentially serious adverse events (AEs), including bleeding and perforation [8]. Therefore, an additional aim of these quality indicators is to minimize the risk of AEs.

Endoscopist-related factors affect the quality and outcomes of screening-related colonoscopy. In a recent meta-analysis, we evaluated the relationship between endoscopists' annual procedural volumes and colonoscopy quality and outcomes [9]. We reported that higher annual colonoscopy volumes were associated with higher CIR, in addition to observing suggestive trends toward lower rates of adverse events [9]. However, no clear associations were found between colonoscopy volumes and ADR [9]. Thus, other potentially relevant endoscopist-related factors are likely present, the consideration of which could help further elucidate variation in colonoscopy quality.

The specialty of the colonoscopist has been proposed as a potential endoscopist-related factor that could explain variations in quality [10]. Endoscopist specialty intuitively makes sense as a potential mechanism for variation, as many have previously highlighted and questioned differences in formal colonoscopy training between specialties [11-13]. Some studies have demonstrated associations between endoscopist specialty and the development of PCCRC $[14,15]$. However, no synthesized evidence is available on this subject. Thus, we propose a systematic review and meta-analysis in order to elucidate whether the specialty of the endoscopist is associated with either colonoscopy quality indicators or procedural outcomes.

\section{Methods}

\section{Overview and Objectives}

Our study will be carried out according to the Preferred Reporting Items for Systematic Reviews and MetaAnalyses (PRISMA) and Meta-analysis Of Observational Studies in Epidemiology (MOOSE) guidelines $[16,17]$. Our review is registered on PROSPERO (CRD42021226251).

The overall objective of this review is to determine whether there is an association between endoscopist specialty and colonoscopy quality metrics and/or outcomes when performed in adult patients. The primary objective is to determine whether there is an association between endoscopist specialty and ADR. The secondary objectives will be to determine whether this association exists for any other established colonoscopy quality metric (including withdrawal time [WT] or CIR) or for any colonoscopy adverse event (including bleeding, perforation, or post-colonoscopy colorectal cancer rate [PCCRC]). No research ethics approval is required for this study given there are no patient-level data being collected. 


\section{Search Strategy}

A detailed electronic search strategy has been designed by the study authors in consultation with a dedicated health research librarian. The electronic databases MEDLINE (Ovid), PubMed, CINAHL, EMBASE, Scopus, Web of Science, and Evidence Based Medicine (EBM) Reviews will be queried from inception of each database to the search date(s). An unabridged search strategy is provided in the Supplementary Materials. Eligibility Criteria

A study will be included for analysis if it meets ALL of the following inclusion criteria:

1. it is an observational study (prospective or retrospective) or clinical trial;

2. it is written in English;

3. it was published in or after the year 2000;

4. it was conducted in adult patients $\geq 18$ years of age undergoing colonoscopy;

5. it reports a direct comparison between at least two separate endoscopist specialties; and

6. it reports one or more of the following colonoscopy quality indicators or outcomes: ADR, CIR, WT, overall or specific AEs, or PCCRC.

A study will be excluded if it meets ANY of the following exclusion criteria:

1. it reports on shared data (on a patient level or endoscopist level) from another study - in these instances, the largest study will be included;

2. it does not report endoscopist specialty data;

3. it reports trainee performance data (from fellows, residents or medical students) in a non-stratified cohort; or

4. it reports data in a non-stratified cohort comprised of upper endoscopic procedures, flexible sigmoidoscopy, or pancreatico-biliary endoscopic procedures.

The eligibility criteria are summarized in Table 1. 
Table 1

Eligibility criteria for study inclusion.

Inclusion criteria

(ALL required)

- original data

- observational study (prospective or

retrospective) or randomized controlled trial

- publication date 2000 or later

- conducted in patients 18 years of age or older

- study contains data on at least two endoscopist specialties

- study reports on at least one colonoscopy quality indicator or adverse event outcome
Exclusion criteria

(ANY required)

- non-English publication

- data overlaps with data from another study (in part or in whole)

- study reports on trainee performance data in a non-stratified cohort

- study reports on endoscopic procedures other than colonoscopy in a non-stratified cohort

\section{Study Selection and Data Abstraction}

All citations will be imported into Rayyan (M Ouzzani, Qatar Computing Research Institute, HBKU, Doha, Qatar) and duplicate citations will be consolidated. Two reviewers (MM, AM) will independently screen all captured titles and abstracts in parallel to identify citations for the subsequent stage (full-text review). All full texts will then be reviewed in parallel by the same reviewers. Any conflicts from the initial and full-text screens will be resolved by a third investigator (NF).

A standardized data abstraction form will be created in order to capture pre-specified data from each study included after the full-text review stage. This form will include relevant study characteristics, patient demographics, endoscopist characteristics, and outcomes of interest. Study-specific definitions of outcomes will also be captured. Endoscopist specialty will be carefully designated as accurately as possible as described by individual studies, and these definitions will be noted. Potential groups will include gastroenterologists, surgeons (including overall, colorectal, and general surgeons), internists, general practitioners, or other. The abstraction process will be carried out in parallel by two independent reviewers (MM, AM) who will then verify one another's work. Any conflicts in data abstraction will again be resolved by a third investigator (NF). In the event that a study meets eligibility criteria but lacks data required for meta-analysis, two separate attempts (one week apart) will be made to contact corresponding study authors to obtain any missing data.

\section{Risk of Bias}

Two authors (MM, AM) will conduct risk of bias assessments in parallel for all studies included in the final analyses. Assessment of observational studies will be assessed using the ROBINS-I tool [18], with conflicts resolved by consensus with a third investigator.

\section{Statistical Analyses}


We will conduct several DerSimonian and Laird meta-analyses to estimate the pooled incidence rates of individual quality metrics and AEs along with $95 \%$ confidence intervals (Cls). Study data will be included only if outcomes are clearly defined and if outcome definitions are consistent with those used in our previous study assessing the relationship between procedural volume and colonoscopy outcomes [9]. Incidence rates from observational studies and randomized trials will be pooled separately (at no point being combined). To address potential causes of inter-study heterogeneity, we will perform several subgroup analyses in clinically and methodologically relevant groups determined a priori. These are summarized in Table 2 . Sources of heterogeneity will also be tested by performing meta-regression. Subgroup analyses and meta-regression will only be performed if there are a minimum of 4 studies in each group [19].

Table 2

Planned subgroup analyses.

\begin{tabular}{|ll|}
\hline Category & Subgroups \\
\hline ptudy & - Screening population (must meet all criteria listed below) versus non-screening \\
population & $0 \leq 15 \%$ of the cohort outside the age range $40-80 ;$ \\
& $0 \leq 15 \%$ of the cohort inpatients; \\
& $0 \leq 15 \%$ of the cohort having colonoscopy for non-standard-screening-related \\
indications ( $i . e$. investigation of symptoms or anemia, screening in patients with a \\
personal history of inflammatory bowel disease (IBD), polyposis syndrome, or CRC)
\end{tabular}

We will also conduct sensitivity analyses where studies of varying quality as per the ROBINS-I tool will be considered separately. Inter-study heterogeneity will be assessed by calculating and reporting the Cochrane $P^{2}$ statistic. Publication bias will be assessed by visual inspection of funnel plots as well as Egger's and Begg's tests [20, 21]. Revman 5.1 (Cochrane Collaboration) and Stata 14.0 (StataCorp) will be the software packages used for statistical analyses. The overall strength of the body of evidence for each outcome will be assessed using the Grading of Recommendations, Assessment, Development and Evaluation (GRADE) framework [22]. 


\section{Discussion}

This systematic review and meta-analysis will provide the first evidence synthesis on whether the specialty of the endoscopist is associated with colonoscopy quality metrics and/or outcomes. Given the established inter-endoscopist quality variations seen with colonoscopy, the results of this study are likely to guide aspects of training, maintenance of certification, credentialing, and quality assurance going forward.

The field of colonoscopy quality is relatively well established, with a number of gastroenterology societies world-wide having endorsed minimum and aspirational benchmarks for several quality indicators and outcomes $[23,24]$. These benchmarks were designed based on available evidence at the time and are intended to reduce endoscopist-level variation and consequently improve patient outcomes. However, significant variation persists in the quality of colonoscopy, an integral procedure in the overall effort to screen for and reduce the burden of CRC [3]. Thus, our study represents an important piece of the puzzle in terms of determining potential endoscopist level factors that are associated with quality metrics and outcomes.

Though our study protocol was designed to mitigate potential biases, our study will nevertheless have limitations. Firstly, given our experience performing meta-analysis on endoscopist factors in colonoscopy [9], we anticipate that several studies will not report potentially relevant confounding variables such as endoscopist age, cumulative experience, procedural volumes, procedural indications, doses of sedating medication, or bowel preparation scores. Furthermore, even if studies do report potential confounders, it is anticipated that most studies assessing our topic of interest will report these data at the study level (rather than at the endoscopist level). Thus, the results of any potential subgroup analyses or metaregression analyses could be misleading given the potential for ecological bias [25]. Additionally, there may be an element of selection bias within each study, whereby surgeons could conceivably have different case mixes than gastroenterologists; this could conceivably bias the results in favour of one group or another when one considers quality indicators such as ADR or adverse event outcomes such as perforation. To mitigate against this risk, we have set strict a priori criteria that define included study samples as primarily screening populations versus non-screening populations (Table 2).

In a broader context, we also anticipate that the relevance and actionability of our study findings will be questioned. Especially when one considers the shift toward competency-based medical education in general [26], and in endoscopic training in particular [27], the current comparisons between gastroenterologists, surgeons, and other endoscopists may become less relevant over time. However, we maintain that this topic is of urgent importance to the current landscape as it pertains to colonoscopy practice. Nevertheless, the practice of audit and feedback of quality metrics as well as educational interventions for independent colonoscopy practitioners are more relevant now than ever, having both been shown to be effective in improving colonoscopy quality $[28,29]$.

Overall, despite these limitations, we anticipate that our study will yield important information on endoscopist specialty and its association with colonoscopy quality indicators and outcomes. Our results 
have potential implications regarding not only direct patient care, but credentialing and training. Our study will summarize the existing state of evidence on this topic, and in so doing, will also serve to elucidate potential improvements for future studies assessing endoscopist level characteristics in the performance of colonoscopy.

\section{Abbreviations}

$\mathrm{AE}$ adverse event

ADR adenoma detection rate

$\mathrm{Cl}$ confidence intervals

CIR cecal intubation rate

CRC colorectal cancer

GRADE Grading of Recommendations, Assessment, Development and Evaluation

MOOSE Meta-analysis Of Observational Studies in Epidemiology

PCCRC post-colonoscopy colorectal cancer

PRISMA Preferred Reporting Items for Systematic Reviews and Meta-Analyses

WT withdrawal time

\section{Declarations}

- Ethics approval and consent to participate

- Ethical approval and consent to participate have both been waived for this study given the use of secondary data from previous published articles.

\section{- Consent for publication}

- Not applicable.

\section{- Availability of data and materials}

- Not applicable. Once generated, data will be available through the corresponding author and through anticipated publication of study results.

\section{- Competing interests}

- Nauzer Forbes has received consultancy fees, speaker's honoraria and research funding from Pentax Canada, consultancy fees from Boston Scientific Corp., and consultancy fees from 
Pendopharm Inc. No funding was received to support this study.

- Funding

- This study is funded by Alberta Health Services Digestive Health Strategic Clinical Network ${ }^{T M}$ $\left(\mathrm{DH} \mathrm{SCN}^{\mathrm{TM}}\right)$ Systematic Review Award.

- Authors' contributions

- NF conceived of and designed the study, co-developed the analysis plan, and drafted the article. DRB contributed to study design and co-developed the analysis plan. YR and MM co-developed the analysis plan. SJH helped design the study. All authors are responsible for critical revision of the article for intellectual content, and final approval of this manuscript.

\section{- Acknowledgements}

- Not applicable.

\section{References}

1. Siegel RL, Miller KD, Goding Sauer A, Fedewa SA, Butterly LF, Anderson JC, Cercek A, Smith RA, Jemal A: Colorectal cancer statistics, 2020. CA: a cancer journal for clinicians 2020, 70(3):145-164.

2. Levin TR, Corley DA, Jensen CD, Schottinger JE, Quinn VP, Zauber AG, Lee JK, Zhao WK, Udaltsova N, Ghai NR et al: Effects of Organized Colorectal Cancer Screening on Cancer Incidence and Mortality in a Large Community-Based Population. Gastroenterology 2018, 155(5):1383-1391 e1385.

3. Nishihara R, Wu K, Lochhead P, Morikawa T, Liao X, Qian ZR, Inamura K, Kim SA, Kuchiba A, Yamauchi $\mathrm{M}$ et al: Long-term colorectal-cancer incidence and mortality after lower endoscopy. The New England journal of medicine 2013, 369(12):1095-1105.

4. Hilsden RJ, Rose SM, Dube C, Rostom A, Bridges R, McGregor SE, Brenner DR, Heitman SJ: Defining and Applying Locally Relevant Benchmarks for the Adenoma Detection Rate. The American journal of gastroenterology 2019, 114(8):1315-1321.

5. Adler A, Wegscheider K, Lieberman D, Aminalai A, Aschenbeck J, Drossel R, Mayr M, Mross M, Scheel $\mathrm{M}$, Schroder $\mathrm{A}$ et al: Factors determining the quality of screening colonoscopy: a prospective study on adenoma detection rates, from 12,134 examinations (Berlin colonoscopy project 3, BECOP-3). Gut 2013, 62(2):236-241.

6. Rex DK, Schoenfeld PS, Cohen J, Pike IM, Adler DG, Fennerty MB, Lieb JG, 2nd, Park WG, Rizk MK, Sawhney MS et al: Quality indicators for colonoscopy. Gastrointestinal endoscopy 2015, 81(1):3153.

7. Kaminski MF, Thomas-Gibson S, Bugajski M, Bretthauer M, Rees CJ, Dekker E, Hoff G, Jover R, Suchanek S, Ferlitsch $\mathrm{M}$ et al: Performance measures for lower gastrointestinal endoscopy: a European Society of Gastrointestinal Endoscopy (ESGE) quality improvement initiative. United European gastroenterology journal 2017, 5(3):309-334. 
8. Rabeneck L, Paszat LF, Hilsden RJ, Saskin R, Leddin D, Grunfeld E, Wai E, Goldwasser M, Sutradhar R, Stukel TA: Bleeding and perforation after outpatient colonoscopy and their risk factors in usual clinical practice. Gastroenterology 2008, 135(6):1899-1906, 1906.e1891.

9. Forbes N, Boyne DJ, Mazurek MS, Hilsden RJ, Sutherland RL, Pader J, Ruan Y, Shaheen AA, Wong C, Lamidi $\mathrm{M}$ et al: Association Between Endoscopist Annual Procedure Volume and Colonoscopy Quality: Systematic Review and Meta-analysis. Clinical gastroenterology and hepatology 2020, 18(10):2192-2208.e2112.

10. Rabeneck L, Paszat LF, Saskin R: Endoscopist Specialty Is Associated With Incident Colorectal Cancer After a Negative Colonoscopy. Clinical gastroenterology and hepatology 2010, 8(3):275-279.

11. Bittner JGt, Marks JM, Dunkin BJ, Richards WO, Onders RP, Mellinger JD: Resident training in flexible gastrointestinal endoscopy: a review of current issues and options. Journal of surgical education 2007, 64(6):399-409.

12. Lee SH, Chung IK, Kim SJ, Kim JO, Ko BM, Hwangbo Y, Kim WH, Park DH, Lee SK, Park CH et al: An adequate level of training for technical competence in screening and diagnostic colonoscopy: a prospective multicenter evaluation of the learning curve. Gastrointestinal endoscopy 2008, 67(4):683-689.

13. Forbes N, Mohamed R, Raman M: Learning curve for endoscopy training: Is it all about numbers? Best practice \& research clinical gastroenterology 2016, 30(3):349-356.

14. Baxter NN, Sutradhar R, Forbes SS, Paszat LF, Saskin R, Rabeneck L: Analysis of administrative data finds endoscopist quality measures associated with postcolonoscopy colorectal cancer. Gastroenterology 2011, 140(1):65-72.

15. Singh H, Nugent Z, Mahmud SM, Demers AA, Bernstein CN. Predictors of colorectal cancer after negative colonoscopy: a population-based study. American journal of gastroenterology 2010;105(3):663-73; quiz 674.

16. Moher D, Shamseer L, Clarke M, Ghersi D, Liberati A, Petticrew M, Shekelle P, Stewart LA: Preferred reporting items for systematic review and meta-analysis protocols (PRISMA-P) 2015 statement. Systematic reviews 2015;4:1.

17. Stroup DF, Berlin JA, Morton SC, Olkin I, Williamson GD, Rennie D, Moher D, Becker BJ, Sipe TA, Thacker SB: Meta-analysis of observational studies in epidemiology: a proposal for reporting. Metaanalysis Of Observational Studies in Epidemiology (MOOSE) group. Journal of the American Medical Association 2000;283(15):2008-2012.

18. Sterne J, Hernan M, Reeves B, Savovic J, Berkman N, Viswanathan M: ROBINS-I: a tool for assessing risk of bias in non-randomised studies of interventions. British medical journal 2016;355:i4919.

19. Fu R, Gartlehner G, Grant M, Shamliyan T, Sedrakyan A, Wilt TJ, Griffith L, Oremus M, Raina P, Ismaila A, et al: Conducting quantitative synthesis when comparing medical interventions: AHRQ and the Effective Health Care Program. Journal of clinical epidemiology 2011;64(11):1187-1197.

20. Egger M, Davey Smith G, Schneider M, Minder C: Bias in meta-analysis detected by a simple, graphical test. British medical journal 1997;315(7109):629-634. 
21. Begg CB, Mazumdar M: Operating characteristics of a rank correlation test for publication bias. Biometrics 1994;50(4):1088-1101.

22. Guyatt GH, Oxman AD, Vist GE, Kunz R, Falck-Ytter Y, Alonso-Coello P, Schunemann HJ: GRADE: an emerging consensus on rating quality of evidence and strength of recommendations. British medical journal 2008;336(7650):924-926.

23. Rex DK, Schoenfeld PS, Cohen J, Pike IM, Adler DG, Fennerty MB, Lieb JG, 2nd, Park WG, Rizk MK, Sawhney MS et al: Quality indicators for colonoscopy. American journal of gastroenterology 2015;110(1):72-90.

24. Rees CJ, Thomas Gibson S, Rutter MD, Baragwanath P, Pullan R, Feeney M, Haslam N: UK key performance indicators and quality assurance standards for colonoscopy. Gut 2016;65(12):19231929.

25. Piantadosi S, Byar DP, Green SB. The ecological fallacy. American journal of epidemiology 1988;127(5):893-904.

26. Frank JR, Snell LS, Cate OT, Holmboe ES, Carraccio C, Swing SR, Harris P, Glasgow NJ, Campbell C, Dath $\mathrm{D}$, et al: Competency-based medical education: theory to practice. Medical teacher 2010;32(8):638-645.

27. Patel SG, Keswani R, Elta G, Saini S, Menard-Katcher P, Del Valle J, Hosford L, Myers A, Ahnen D, Schoenfeld P: Status of Competency-Based Medical Education in Endoscopy Training: A Nationwide Survey of US ACGME-Accredited Gastroenterology Training Programs. American journal of gastroenterology 2015;110(7):956-962.

28. Bishay K, Causada-Calo N, Scaffidi MA, Walsh CM, Anderson JT, Rostom A, Dube C, Keswani RN, Heitman SJ, Hilsden RJ, et al: Associations between endoscopist feedback and improvements in colonoscopy quality indicators: a systematic review and meta-analysis. Gastrointestinal endoscopy 2020;92(5):1030-1040.e1039.

29. Causada-Calo NS, Gonzalez-Moreno El, Bishay K, Shorr R, Dube C, Heitman SJ, Hilsden RJ, Rostom A, Walsh C, Anderson JT, et al: Educational interventions are associated with improvements in colonoscopy quality indicators: a systematic review and meta-analysis. Endoscopy international open 2020;8(10):E1321-1331. 